\title{
Global Dynamic Behavior of a Multigroup Cholera Model with Indirect Transmission
}

\author{
Ming-Tao Li, ${ }^{1}$ Gui-Quan Sun, ${ }^{2,3,4}$ Juan Zhang, ${ }^{1}$ and Zhen Jin ${ }^{1,2}$ \\ ${ }^{1}$ Department of Mathematics, North University of China, Taiyuan, Shan'xi 030051, China \\ ${ }^{2}$ Complex Systems Center, Shanxi University, Taiyuan, Shan'xi 030006, China \\ ${ }^{3}$ Institute of Information Economy, Hangzhou Normal University, Hangzhou, Zhejiang 310036, China \\ ${ }^{4}$ School of Mathematical Sciences, Fudan University, Shanghai 200433, China \\ Correspondence should be addressed to Zhen Jin; jinzhn@263.net
}

Received 4 October 2013; Accepted 7 November 2013

Academic Editor: Antonia Vecchio

Copyright ( 2013 Ming-Tao Li et al. This is an open access article distributed under the Creative Commons Attribution License, which permits unrestricted use, distribution, and reproduction in any medium, provided the original work is properly cited.

\begin{abstract}
For a multigroup cholera model with indirect transmission, the infection for a susceptible person is almost invariably transmitted by drinking contaminated water in which pathogens, $V$. cholerae, are present. The basic reproduction number $\mathscr{R}_{0}$ is identified and global dynamics are completely determined by $\mathscr{R}_{0}$. It shows that $\mathscr{R}_{0}$ is a globally threshold parameter in the sense that if it is less than one, the disease-free equilibrium is globally asymptotically stable; whereas if it is larger than one, there is a unique endemic equilibrium which is global asymptotically stable. For the proof of global stability with the disease-free equilibrium, we use the comparison principle; and for the endemic equilibrium we use the classical method of Lyapunov function and the graph-theoretic approach.
\end{abstract}

\section{Introduction}

Cholera, a waterborne gastroenteric infection, remains a significant threat to public health in the developing world. Outbreaks of cholera occur cyclically, usually twice per year in endemic areas, and the intensity of these outbreaks varies over longer periods [1]. Hence, in the last few decades, enormous attention is being paid to the cholera disease and several mathematical dynamic models have been developed to study the transmission of cholera [1-7]. In these papers, they consider the population is uniformly mixed, but many factors can lead to heterogeneity in a host population. So in this paper we divide different population into different groups, which can be divided geographically into communities, cities, and countries, to incorporate differential infectivity of multiple strains of the disease agent.

In the case of cholera, the transmission usually occurs through ingestion of contaminated water or feces rather than through casual human-human contact [1]. Therefore, direct contact of healthy person with an infected person is not a risk for contracting infection, whereas a healthy person may contract infection by drinking contaminated water in which pathogens, $V$. cholerae, are present [2]. The members of this bacterial genus ( $V$. cholerae) naturally colonize in lakes, rivers, and estuaries. Therefore we consider that cholera transmits to other individuals via bacteria in the aquatic environment and formulates a multi-group epidemic model for cholera. Let $N$ be the total population which is divided into four epidemiological compartments, susceptible compartment $S$, infectious compartment $I$, recovered compartment $R$, and vaccinated compartment $V$. Let $W$ be the density of $V$. cholerae in the aquatic environment. As a consequence of the increase in the density of virulent $V$. cholerae in the aquatic environment, humans become infected and begin to shed increasing numbers of bacteria into the aquatic environment, further elevating bacterial density and exacerbating the outbreak [1]. The growth rate of density of bacteria in the aquatic environment is assumed to be proportional to the number of infectious individuals. We assume that the immunity induced by vaccination is perfect; therefore individuals in vaccinated individuals $V$ cannot be infected. The model is called a multi-group cholera SIRVW epidemic model.

In recent years, multi-group epidemic models have been used to describe the transmission dynamics of many 
infectious disease in heterogeneous individuals, such as HIV/AIDS [8], dengue [9], West-Nile virus [10], sexually transmitted diseases [11]; and so on. It is well known that global dynamics of multi-group models with higher dimensions, especially the global stability of the endemic equilibrium, is a very challenging problem. Lajmanovich and York [12] proved global stability of the unique endemic equilibrium by using a quadratic global Lyapunov function on a class of $n$-group SIS models for gonorrhea; Hethcote [13] proved global stability of the endemic equilibrium for multi-group SIR model without vital dynamics; Thieme [14] proved global stability of the endemic equilibrium of multigroup SEIRS model under certain restrictions. However, they only proved global stability of the endemic equilibrium for multi-group model under some special conditions. In 2006, Guo et al. [15] have first succeeded to establish the complete global dynamics for a multi-group SIR model, by making use of the theory of non-negative matrices, Lyapunov functions and a subtle grouping technique in estimating the derivatives of Lyapunov functions guided by graph theory. By using the results or ideas of [15], the papers [16, 17] proved the global stability of the endemic equilibrium for multi-group model with nonlinear incidence rates and the papers $[18,19]$ proved the global stability of the endemic equilibrium for multigroup model with distributed delays.

Distinguishing from these multi-group models with direct transmission from person to person, a multi-group cholera model with indirect transmission from the bacteria of the aquatic environment to person is proposed in this paper. We prove that the disease-free equilibrium is globally asymptotically stable if $\mathscr{R}_{0}<1$, while an endemic equilibrium exists uniquely and is globally asymptotically stable if $\mathscr{R}_{0}>1$.

The organization of this paper is as follows. In Section 2, we construct a multi-group cholera epidemiological and give some dynamic analysis on the disease-free equilibrium and the endemic equilibrium. An example is given in Section 3 and some conclusions are included in Section 4.

\section{Mathematical Modeling and Analysis}

For a multi-group epidemic model with cholera, the population of human is divided into $n$ discrete groups, where $n \in$ $\mathbb{N}$. Let $S_{i}(t), I_{i}(t), R_{i}(t)$, and $V_{i}(t)$ be the numbers of susceptible, infectious, recovered, and vaccinated individuals in group $i=1,2, \ldots, n$ at time $t$, respectively. Let $W_{i}(t)$ be the density of bacteria in the aquatic environment in group $i=1,2, \ldots, n$ at time $t$. Based on the assumptions in Section 1, the disease transmission rate of cholera between compartments $S_{i}$ and $W_{j}$ is denoted by $\beta_{i j}$, which means the susceptible individuals in the $i$ th group can contact the bacteria of the aquatic environment in the $j$ th $(j=$ $1,2, \ldots, n)$ group. So the new infection that occurred in the $i$ th group is given by $\sum_{j=1}^{n} \beta_{i j} S_{i} W_{j}$. The recruitment rate of individuals into $S_{i}(t)$ compartment with the $i$ th group is given by a constant $A_{i}$. Within the $i$ th group, it is assumed that natural death of human is $d_{i}$. A simple immunization policy is considered where the vaccination rate in $S_{i}(t)$ compartment is given by a constant $\gamma_{i}$ and the losing immunity rate from vaccination individuals is $\lambda_{i}$. We assume that individuals in $I_{i}(t)$ compartment recover with a rate constant $r_{i}$. In $W_{i}(t)$ compartment, the brucella shedding rate from $I_{i}(t)$ compartment is $k_{i}$, and the decaying rate of brucella is $\delta_{i}$. So a general multi-group SIRVW epidemic model is described by the following system of differential equations:

$$
\begin{aligned}
& \frac{d S_{i}}{d t}=A_{i}-\left(d_{i}+\gamma_{i}\right) S_{i}+\lambda_{i} V_{i}-\sum_{j=1}^{n} \beta_{i j} S_{i} W_{j}, \\
& \frac{d I_{i}}{d t}=\sum_{j=1}^{n} \beta_{i j} S_{i} W_{j},-\left(d_{i}+r_{i}\right) I_{i}, \\
& \frac{d R_{i}}{d t}=r_{i} I_{i}-d_{i} R_{i}, \\
& \frac{d V_{i}}{d t}=\gamma_{i} S_{i}-\left(\lambda_{i}+d_{i}\right) V_{i}, \\
& \frac{d W_{i}}{d t}=k_{i} I_{i}-\delta_{i} W_{i}, \quad \quad i=1,2, \ldots, n .
\end{aligned}
$$

The parameters $A_{i}, d_{i}, \lambda_{i}, \gamma_{i}, k_{i}$, and $\delta_{i}$ are positive for all $i=1,2, \ldots, n$, which is made for the biological justification. And we assume that $\beta_{i j}$ is nonnegative for all $i, j=$ $1,2, \ldots, n$ and $n$-square matrix $B=\left(\beta_{i j}\right)_{1 \leq i, j \leq n}$ is irreducible, which implies that every pair of groups is joined by an infectious path so that the presence of an infectious individual in the first group can cause infection in the second group.

Observe that the variable $R_{i}$ does not appear in the first and last two equations of system (1); this allows us to consider the following reduced system:

$$
\begin{aligned}
& \frac{d S_{i}}{d t}=A_{i}-\left(d_{i}+\gamma_{i}\right) S_{i}+\lambda_{i} V_{i} \\
& \frac{d I_{i}}{d t}=\sum_{j=1}^{n} \beta_{i j} S_{i} W_{j}-\left(d_{i}+r_{i}\right) I_{i}, \\
& \frac{d V_{i}}{d t}=\gamma_{i} S_{i}-\left(\lambda_{i}+d_{i}\right) V_{i}, \\
& \frac{d W_{i}}{d t}=k_{i} I_{i}-\delta_{i} W_{i},
\end{aligned}
$$

$$
i=1,2, \ldots, n \text {. }
$$

For each group $i$, adding the four equations in system (2) gives

$$
\begin{aligned}
\frac{d\left(S_{i}+I_{i}+V_{i}\right)}{d t} & =A_{i}-d_{i}\left(S_{i}+I_{i}+V_{i}\right)-r_{i} I_{i} \\
& \leq\left(A_{i}-d_{i}\left(S_{i}+I_{i}+V_{i}\right)\right),
\end{aligned}
$$


then it follows that

$$
\lim _{t \rightarrow \infty} \sup \left(S_{i}+I_{i}+V_{i}\right) \leq \frac{A_{i}}{d_{i}}, \quad \lim _{t \rightarrow \infty} \sup W_{i} \leq \frac{k_{i} A_{i}}{d_{i} \delta_{i}}
$$

Therefore, omega limit sets of system (2) are contained in the following bounded region in the nonnegative cone of $\mathbb{R}^{4 n}$ :

$$
\begin{gathered}
X=\left\{\left(S_{i}, I_{i}, V_{i}, W_{i}\right) \mid S_{i}, I_{i}, V_{i}, W_{i} \geq 0,0 \leq\left(S_{i}+I_{i}+V_{i}\right)\right. \\
\left.\leq \frac{A_{i}}{d_{i}}, W_{i} \leq \frac{k_{i} A_{i}}{d_{i} \delta_{i}}, i=1,2, \ldots, n\right\} .
\end{gathered}
$$

It can be verified that region $X$ is positively invariant with respect to system (2). System (2) always has a disease-free equilibrium

$$
P_{0}=\left(S_{1}^{0}, 0, V_{1}^{0}, 0, \ldots, S_{i}^{0}, 0, V_{i}^{0}, 0, \ldots, S_{n}^{0}, 0, V_{n}^{0}, 0\right)
$$

on the boundary of $X$, where

$$
S_{i}^{0}=\frac{A_{i}\left(\lambda_{i}+d_{i}\right)}{d_{i}\left(\lambda_{i}+d_{i}+\gamma_{i}\right)}, \quad V_{i}^{0}=\frac{A_{i} \gamma_{i}}{d_{i}\left(\lambda_{i}+d_{i}+\gamma_{i}\right)} .
$$

2.1. The Basic Reproduction Number. According to the next generation matrix formulated in papers [20-22], we define the basic reproduction number $\mathscr{R}_{0}$ of system (2). In order to formulate $\mathscr{R}_{0}$, we order the infected variables first by disease state and then by group, that is,

$$
I_{1}, W_{1}, I_{2}, W_{2}, \ldots, I_{n}, W_{n}
$$

Consider the following auxiliary system:

$$
\begin{array}{r}
\frac{d I_{i}}{d t}=\sum_{j=1}^{n} \beta_{i j} S_{i} W_{j}-\left(d_{i}+r_{i}\right) I_{i}, \\
\frac{d W_{i}}{d t}=k_{i} I_{i}-\delta_{i} W_{i}, \\
i=1,2, \ldots, n .
\end{array}
$$

Follow the recipe from van den Driessche and Watmough [21] to obtain

$$
F=\left(\begin{array}{ccccccc}
0 & \beta_{11} S_{1}^{0} & 0 & \beta_{12} S_{1}^{0} & \cdots & 0 & \beta_{1 n} S_{1}^{0} \\
0 & 0 & 0 & 0 & \cdots & 0 & 0 \\
0 & \beta_{21} S_{2}^{0} & 0 & \beta_{22} S_{2}^{0} & \cdots & 0 & \beta_{2 n} S_{2}^{0} \\
0 & 0 & 0 & 0 & \cdots & 0 & 0 \\
\vdots & \vdots & \vdots & \vdots & & \vdots & \vdots \\
0 & \beta_{n 1} S_{n}^{0} & 0 & \beta_{n 2} S_{n}^{0} & \cdots & 0 & \beta_{n n} S_{n}^{0} \\
0 & 0 & 0 & 0 & \cdots & 0 & 0
\end{array}\right)_{2 n \times 2 n},
$$

$$
V=\left(\begin{array}{ccccccc}
d_{1}+r_{1} & 0 & 0 & 0 & \cdots & 0 & 0 \\
-k_{1} & \delta_{1} & 0 & 0 & \cdots & 0 & 0 \\
0 & 0 & d_{2}+r_{2} & 0 & \cdots & 0 & 0 \\
0 & 0 & -k_{2} & \delta_{2} & \cdots & 0 & 0 \\
\vdots & \vdots & \vdots & \vdots & & \vdots & \vdots \\
0 & 0 & 0 & 0 & \cdots & d_{n}+r_{n} & 0 \\
0 & 0 & 0 & 0 & \cdots & -k_{1} & \delta_{n}
\end{array}\right)_{2 n \times 2 n}
$$

We can get the inverse of $V$, which equals

$$
V^{-1}=\left(\begin{array}{ccccccc}
\frac{1}{d_{1}+r_{1}} & 0 & 0 & 0 & \cdots & 0 & 0 \\
\frac{k_{1}}{\delta_{1}\left(d_{1}+r_{1}\right)} & \frac{1}{\delta_{1}} & 0 & 0 & \cdots & 0 & 0 \\
0 & 0 & \frac{1}{d_{2}+r_{2}} & 0 & \cdots & 0 & 0 \\
0 & 0 & \frac{k_{2}}{\delta_{2}\left(d_{2}+r_{2}\right)} & \frac{1}{\delta_{2}} & \cdots & 0 & 0 \\
\vdots & \vdots & \vdots & \vdots & & \vdots & \vdots \\
0 & 0 & 0 & 0 & \cdots & \frac{1}{d_{n}+r_{n}} & 0 \\
0 & 0 & 0 & 0 & \cdots & \frac{k_{n}}{\delta_{n}\left(d_{n}+r_{n}\right)} & \frac{1}{\delta_{n}}
\end{array}\right)_{2 n \times 2 n}
$$


Thus, the next generation matrix is $F V^{-1}$,

$$
\begin{aligned}
F V^{-1}= & \left(\begin{array}{cccccc}
A_{11} & \cdots & A_{1 n} & B_{11} & \cdots & B_{1 n} \\
\vdots & & \vdots & \vdots & & \vdots \\
A_{n 1} & \cdots & A_{n n} & B_{n 1} & \cdots & B_{n n} \\
0 & \cdots & 0 & 0 & \cdots & 0 \\
\vdots & & \vdots & \vdots & & \vdots \\
0 & \cdots & 0 & 0 & \cdots & 0
\end{array}\right)_{2 n \times 2 n}, \\
A & =\left(\begin{array}{cccc}
A_{11} & A_{12} & \cdots & A_{1 n} \\
A_{21} & A_{22} & \cdots & A_{2 n} \\
\vdots & \vdots & & \vdots \\
A_{n 1} & A_{n 2} & \cdots & A_{n n}
\end{array}\right)_{n \times n} .
\end{aligned}
$$

So we can calculate the basic reproduction number of system (2),

$$
\mathscr{R}_{0}=\rho\left(F V^{-1}\right)=\rho(A),
$$

where

$$
\begin{array}{r}
A_{i j}=\frac{\beta_{i j} k_{j} S_{i}^{0}}{\delta_{j}\left(d_{j}+r_{j}\right)}, \quad S_{i}^{0}=\frac{A_{i}\left(\lambda_{i}+d_{i}\right)}{d_{i}\left(\lambda_{i}+d_{i}+\gamma_{i}\right)}, \\
i=1,2, \ldots, n,
\end{array}
$$

$$
J_{4}=\left(\begin{array}{ccc}
-\left(d_{1}+\gamma_{1}\right) & \lambda_{1} & 0 \\
\gamma_{1} & -\left(d_{1}+\lambda_{1}\right) & 0 \\
0 & 0 & -\left(d_{2}+\gamma_{2}\right) \\
0 & 0 & \gamma_{2} \\
\vdots & \vdots & \vdots \\
0 & 0 & 0 \\
0 & 0 & 0
\end{array}\right.
$$

Calculate the eigenvalues of $J_{4}$ :

$$
\begin{gathered}
s\left(J_{4}\right)=\max \left\{-d_{1}, \ldots,-d_{n},-\left(d_{1}+\lambda_{1}+\gamma_{1}\right), \ldots,\right. \\
\left.-\left(d_{n}+\lambda_{n}+\gamma_{n}\right)\right\}<0 .
\end{gathered}
$$

If $\mathscr{R}_{0}<1$, then $s(M)<0$ and $s\left(\left.J\right|_{P_{0}}\right)<0$, and the diseasefree equilibrium $P_{0}$ of (2) is locally asymptotically stable.

Now we will prove that the disease-free equilibrium $P_{0}$ of system (2) is globally attractive when $\mathscr{R}_{0}<1$. From the third equation of system (2), we have

$$
\begin{aligned}
\frac{d V_{i}}{d t} & =\gamma_{i} S_{i}-\left(\lambda_{i}+d_{i}\right) V_{i} \\
& =\gamma_{i}\left(N_{i}-\left(I_{i}+V_{i}\right)\right)-\left(\lambda_{i}+d_{i}\right) V_{i} \\
& \leq \gamma_{i} \frac{A_{i}}{d_{i}}-\left(\lambda_{i}+\gamma_{i}+d_{i}\right) V_{i} .
\end{aligned}
$$

and $\rho$ denotes the spectral radius. As we will show, $\mathscr{R}_{0}$ is the key threshold parameters whose values completely characterize the global dynamics of system (2).

\subsection{Global Stability of the Disease-Free Equilibrium of System} (2). For the disease-free equilibrium $P_{0}$ of system (2), we have the following property.

Theorem 1. If $\mathscr{R}_{0}<1$, the disease-free equilibrium $P_{0}$ of system (2) is globally asymptotically stable in the region $X$.

Proof. Let $M=F-V$, and define $s(M)=\max \{\operatorname{Re} \lambda: \lambda$ is an eigenvalue of $M\}$, so $s(M)$ is a simple eigenvalue of $M$ with a positive eigenvector [23]. By Theorem 2 in [21], there hold two equivalences:

$$
\mathscr{R}_{0}>1 \Longleftrightarrow s(M)>0, \quad \mathscr{R}_{0}<1 \Longleftrightarrow s(M)<0 .
$$

To prove the locally stability of disease-free equilibrium, we check the hypotheses (A1)-(A5) in [21]. Hypotheses (A1)(A4) are easily verified, while (A5) is satisfied if all eigenvalues of the $4 n \times 4 n$ matrix

$$
\left.J\right|_{P_{0}}=\left(\begin{array}{cc}
M & 0 \\
J_{3} & J_{4}
\end{array}\right)_{4 n \times 4 n}
$$

have negative real parts, where $J_{3}=-F$,

$$
\left.\begin{array}{cccc}
0 & \cdots & 0 & 0 \\
0 & \cdots & 0 & 0 \\
\lambda_{2} & \cdots & 0 & 0 \\
-\left(d_{2}+\lambda_{2}\right) & \cdots & 0 & 0 \\
\vdots & & \vdots & \vdots \\
0 & \cdots & -\left(d_{n}+\gamma_{n}\right) & \lambda_{n} \\
0 & \cdots & \gamma_{n} & -\left(d_{n}+\lambda_{n}\right)
\end{array}\right)_{2 n \times 2 n}
$$

So we can have that, for a small enough positive number $\epsilon_{1}$, there exists $t_{i}>0, i=1,2, \ldots, n$ such that for all $t>t_{i}$,

$$
V_{i} \leq \frac{A_{i} \gamma_{i}}{d_{i}\left(\lambda_{i}+\gamma_{i}+d_{i}\right)}+\epsilon_{1}=V_{i}^{0}+\epsilon_{1}
$$

Also from the equations of system (2), we have

$$
\begin{aligned}
\frac{d S_{i}}{d t} & =A_{i}+\lambda_{i} V_{i}-\left(\gamma_{i}+d_{i}\right) S_{i}-\sum_{j=1}^{n} \beta_{i j} S_{i} W_{j} \\
& \leq A_{i}+\lambda_{i}\left(V_{i}^{0}+\epsilon_{1}\right)-\left(d_{i}+\gamma_{i}\right) S_{o} .
\end{aligned}
$$

Then

$$
\lim _{t \rightarrow \infty} \sup _{i}=\frac{A_{i}+\lambda_{i}\left(V_{i}^{0}+\epsilon_{1}\right)}{d_{i}+\gamma_{i}}=S_{i}^{0}+\epsilon_{2}, \quad\left(\epsilon_{2}=\frac{\lambda_{i} \epsilon_{1}}{d_{i}+\gamma_{i}}\right) .
$$


From system (9) and $S_{i} \leq S_{i}^{0}+\epsilon_{2}$ with all $t>t_{i}$. Thus, when $t>t_{i}$, we derive

$$
\begin{aligned}
& \frac{d I_{i}}{d t}=\left(S_{i}^{0}+\epsilon_{2}\right) \sum_{j=1}^{n} \beta_{i j} W_{j}-\left(d_{i}+r_{i}\right) I_{i}, \\
& \frac{d W_{i}}{d t}=k_{i} I_{i}-\delta_{i} W_{i}, \\
& \quad i=1,2, \ldots, n .
\end{aligned}
$$

Consider the following auxiliary system

$$
\begin{aligned}
& \frac{d I_{i}^{\prime}}{d t}=\left(S_{i}^{0}+\epsilon_{2}\right) \sum_{j=1}^{n} \beta_{i j} W_{j}^{\prime}-\left(d_{i}+r_{i}\right) I_{i}^{\prime}, \\
& \frac{d W_{i}^{\prime}}{d t}=k_{i} I_{i}^{\prime}-\delta_{i} W_{i}^{\prime}, \quad \\
& \quad i=1,2, \ldots, n .
\end{aligned}
$$

Let $M_{0}$ be the matrix defined by

$$
M_{0}=\left(\begin{array}{ccccccc}
0 & \beta_{11} & 0 & \beta_{12} & \cdots & 0 & \beta_{1 n} \\
0 & 0 & 0 & 0 & \cdots & 0 & 0 \\
0 & \beta_{21} & 0 & \beta_{22} & \cdots & 0 & \beta_{2 n} \\
0 & 0 & 0 & 0 & \cdots & 0 & 0 \\
\vdots & \vdots & \vdots & \vdots & & \vdots & \vdots \\
0 & \beta_{n 1} & 0 & \beta_{n 2} & \cdots & 0 & \beta_{n n} \\
0 & 0 & 0 & 0 & \cdots & 0 & 0
\end{array}\right)_{2 n \times 2 n},
$$

and set $M_{1}=M+\epsilon_{2} M_{0}$. It follows from Theorem 2 in [21] that $\mathscr{R}_{0}<1$ if and only if $s(M)<0$. Thus, there exists an $\epsilon_{2}>0$ small enough such that $s\left(M_{1}\right)<0$. Using the Perron-Frobenius theorem, all eigenvalues of the matrix $M_{1}$ have negative real parts when $s\left(M_{1}\right)<0$. Therefore it has

$$
\begin{gathered}
\left(I_{1}^{\prime}(t), W_{1}^{\prime}(t), I_{2}^{\prime}(t), W_{2}^{\prime}(t), \ldots, I_{n}^{\prime}(t), W_{n}^{\prime}(t)\right) \\
\longrightarrow(0,0,0,0, \ldots, 0,0), \quad t \longrightarrow \infty
\end{gathered}
$$

which implies that the zero solution of system (24) is globally asymptotically stable. Using the comparison principle of Smith and Waltman [23], we know that

$$
\begin{gathered}
\left(I_{1}(t), W_{1}(t), I_{2}(t), W_{2}(t), \ldots, I_{n}(t), W_{n}(t)\right) \\
\longrightarrow(0,0,0,0, \ldots, 0,0), \quad t \longrightarrow \infty .
\end{gathered}
$$

By the theory of asymptotic autonomous system of Thieme [24], it is also known that

$$
\begin{aligned}
\left(S_{1}(t)\right. & \left., V_{1}(t), \ldots, S_{n}(t), V_{n}(t)\right) \\
& \longrightarrow\left(S_{1}(0), V_{1}(0), \ldots, S_{n}(0), V_{n}(0)\right), \quad t \longrightarrow \infty .
\end{aligned}
$$

So $P_{0}$ is globally attractive when $\mathscr{R}_{0}<1$. It follows that the disease-free equilibrium $P_{0}$ of (2) is globally asymptotically stable when $\mathscr{R}_{0}<1$. This completes the proof.
2.3. The Uniform Persistence and Unique Positive Solution of System (2). In this section, we give the proof of the uniform persistence and the unique positive solution of system (2). Define

$$
\begin{array}{r}
X_{0}=\left\{\left(S_{i}, I_{i}, V_{i}, W_{i}\right) \in X \mid I_{i}, W_{i}>0, i=1,2, \ldots, n\right\}, \\
\partial X_{0}=X \mid X_{0} .
\end{array}
$$

Theorem 2. When $\mathscr{R}_{0}>1$, there exists a positive constant $\varepsilon_{1}$ such that when $\left\|I_{i}(0)\right\|<\varepsilon_{1},\left\|W_{i}(0)\right\|<\varepsilon_{1}$ for $\left(S_{i}(0)\right.$, $\left.I_{i}(0), V_{i}(0), W_{i}(0)\right) \in X_{0}$

$$
\limsup _{t \rightarrow \infty} \max \left\{I_{i}(t), W_{i}(t)\right\}>\varepsilon_{1}, \quad i=1,2, \ldots, n .
$$

Proof. Consider the following system:

$$
\begin{array}{r}
\frac{d S_{i}}{d t}=A_{i}+\lambda_{i} V_{i}-\left(\gamma_{i}+d_{i}\right) S_{i}, \\
\frac{d V_{i}}{d t}=\gamma_{i} S_{i}-\left(\lambda_{i}+d_{i}\right) V_{i}, \\
i=1,2, \ldots, n .
\end{array}
$$

Using Corollary 3.2 in Zhao and Jing [25], it then follows that system (31) has a unique positive equilibrium $\left(S_{1}^{0}, V_{1}^{0}, \ldots, S_{n}^{0}, V_{n}^{0}\right)$ which is globally asymptotically stable.

As to $\mathscr{R}_{0}>1 \Leftrightarrow s(M)>0$, choose $\varepsilon>0$ small enough such that $s\left(M_{2}\right)>0$, where $M_{2}=M-\varepsilon M_{0}$. Let us consider a perturbed system

$$
\begin{aligned}
& \frac{d S_{i}}{d t}=A_{i}-\left(d_{i}+\gamma_{i}\right) S_{i}+\lambda_{i} V_{i}-\varepsilon_{1} S_{i} \sum_{j=1}^{n} \beta_{i j}, \\
& \frac{d V_{i}}{d t}=\gamma_{i} S_{i}-\left(\lambda_{i}+d_{i}\right) V_{i},
\end{aligned}
$$

$$
i=1,2, \ldots, n .
$$

From our previous analysis of system (32), we can restrict $\varepsilon_{1}>0$ small enough such that (32) admits a unique positive equilibrium $\left(S_{i}^{0}\left(\varepsilon_{1}\right), V_{i}^{0}\left(\varepsilon_{1}\right), i=1,2, \ldots, n\right)$ which is globally asymptotically stable. $S_{i}^{0}\left(\varepsilon_{1}\right)$ is continuous in $\varepsilon_{1}$, so we can further restrict $\varepsilon_{1}$ small enough such that $S_{i}^{0}\left(\varepsilon_{1}\right)>$ $S_{i}^{0}-\varepsilon, i=1,2, \ldots, n$.

For the sake of contradiction of Theorem 2, there is a $T>$ 0 such that $I_{i}(t)<\varepsilon_{1}, W_{i}(t)<\varepsilon_{1}, i=1,2, \ldots, n$, for all $t \geq T$. Then for $t \geq T$, we have

$$
\begin{aligned}
& \frac{d S_{i}}{d t} \geq A_{i}-\left(d_{i}+\gamma_{i}\right) S_{i}+\lambda_{i} R_{i}-\varepsilon_{1} S_{i} \sum_{j=1}^{n} \beta_{i j}, \\
& \frac{d R_{i}}{d t}=\gamma_{i} S_{i}-\left(\lambda_{i}+d_{i}\right) R_{i},
\end{aligned}
$$

$$
i=1,2, \ldots, n .
$$

Since the equilibrium $\left(S_{i}^{0}\left(\varepsilon_{1}\right), V_{i}^{0}\left(\varepsilon_{1}\right), i=1,2, \ldots, n\right)$ of (32) is globally asymptotically stable and $S_{i}^{0}\left(\varepsilon_{1}\right)>S_{i}^{0}-\varepsilon$, 
$i=1,2, \ldots, n$. There exists a $T_{1}>T>0$ such that $S_{i}(t)>$ $S_{i}^{0}-\varepsilon, i=1,2, \ldots, n$ for $t>T_{1}$. As a consequence, for $t>T_{1}$, there holds

$$
\begin{aligned}
& \frac{d I_{i}}{d t} \geq\left(S_{i}^{0}-\varepsilon\right) \sum_{j=1}^{n} \beta_{i j} W_{j}-\left(d_{i}+r_{i}\right) I_{i}, \\
& \frac{d W_{i}}{d t}=k_{i} I_{i}-\delta_{i} W_{i},
\end{aligned}
$$

$$
i=1,2, \ldots, n \text {. }
$$

Consider the following system

$$
\begin{aligned}
& \frac{d I_{i}^{\prime}}{d t}=\left(S_{i}^{0}-\varepsilon\right) \sum_{j=1}^{n} \beta_{i j} W_{j}^{\prime}-\left(d_{i}+r_{i}\right) I_{i}^{\prime}, \\
& \frac{d W_{i}^{\prime}}{d t}=k_{i} I_{i}^{\prime}-\delta_{i} W_{i}^{\prime}, \\
& \quad i=1,2, \ldots, n .
\end{aligned}
$$

Since the matrix $M_{2}$ has positive eigenvalue $s\left(M_{2}\right)$ with a positive eigenvector. It is easy to see that

$$
\begin{aligned}
\left(I_{1}^{\prime}(t),\right. & \left.W_{1}^{\prime}(t), I_{2}^{\prime}(t), W_{2}^{\prime}(t), \ldots, I_{n}^{\prime}(t), W_{n}^{\prime}(t)\right) \\
& \longrightarrow(\infty, \infty, \infty, \infty, \ldots, \infty, \infty), \quad t \longrightarrow \infty
\end{aligned}
$$

Using the comparison principle of Smith and Waltman [23], we also know that

$$
\begin{aligned}
\left(I_{1}(t),\right. & \left.W_{1}(t), I_{2}(t), W_{2}(t), \ldots, I_{n}(t), W_{n}(t)\right) \\
& \longrightarrow(\infty, \infty, \infty, \infty, \ldots, \infty, \infty), \quad t \longrightarrow \infty
\end{aligned}
$$

which leads to a contradiction, therefore we claim that

$$
\limsup _{t \rightarrow \infty} \max \left\{I_{i}(t), W_{i}(t)\right\}>\varepsilon_{1}, \quad i=1,2, \ldots, n .
$$

This completes the proof.

We also have the following result of system (2).

Theorem 3. If $\mathscr{R}_{0}>1$, then system (2) admits at least one positive equilibrium, and there is a positive constant $\varepsilon$ such that every solution $\left(S_{i}(t), I_{i}(t), V_{i}(t), W_{i}(t)\right)$ of the system (2) with $\left(S_{i}(0), I_{i}(0), V_{i}(0), W_{i}(0)\right) \in X_{0}$ satisfies

$$
\min \left\{\liminf _{t \rightarrow \infty} I_{i}(t), \liminf _{t \rightarrow \infty} W_{i}(t)\right\} \geq \varepsilon, \quad i=1,2, \ldots, n,
$$

which implies that system (2) is uniformly persistent.

Proof. Now we prove that system (2) is uniformly persistent with respect to $\left(X_{0}, \partial X_{0}\right)$. By the form of (2), it is easy to see that both $X$ and $X_{0}$ are positively invariant and $\partial X_{0}$ is relatively closed in $X$. Furthermore system (2) is point dissipative. Let

$$
\begin{aligned}
& M_{\partial} \\
& =\left\{\left(S_{i}(0), I_{i}(0), V_{i}(0), W_{i}(0)\right) \mid\left(S_{i}(t), I_{i}(t), V_{i}(t), W_{i}(t)\right)\right. \\
& \left.\quad \in \partial X_{0}, \forall t \geq 0, i=1,2, \ldots, n\right\} .
\end{aligned}
$$

It is easy to show that

$$
M_{\partial}=\left\{\left(S_{i}(t), 0, V_{i}(t), 0\right) \mid S_{i}(t), V_{i}(t) \geq 0, i=1,2, \ldots, n\right\} .
$$

Noting that $\left\{\left(S_{i}(t), 0, V_{i}(t), 0\right) \quad \mid S_{i}(t), V_{i}(t) \geq 0, i=\right.$ $1,2, \ldots, n\} \subseteq M_{\partial}$. We only need to prove $M_{\partial} \subseteq$ $\left\{\left(S_{i}(t), 0, V_{i}(t), 0\right) \quad \mid \quad S_{i}(t), V_{i}(t) \geq 0, i=1,2, \ldots, n\right\}$. Assume $\left(S_{i}(0), I_{i}(0), V_{i}(0), W_{i}(0), i=1,2, \ldots, n\right) \in M_{\partial}$. It suffices to show that $I_{i}(t)=0, W_{i}(t)=0$, for all $t \geq 0$, $i=1,2, \ldots, n$. Suppose not. Then there exist an $i_{0}, 1 \leq i_{0} \leq$ $n$, and $t_{0} \geq 0$ such that $I_{i_{0}}\left(t_{0}\right)>0, W_{i_{0}}\left(t_{0}\right)>0$ and we partition $\{1,2, \ldots, n\}$ into two sets $Q_{1}$ and $Q_{2}$ such that

$$
\begin{aligned}
& \left(I_{i}\left(t_{0}\right), W_{i}\left(t_{0}\right)\right)^{T}=0, \quad \forall i \in Q_{1}, \\
& \left(I_{i}\left(t_{0}\right), W_{i}\left(t_{0}\right)\right)^{T}>0, \quad \forall i \in Q_{2} .
\end{aligned}
$$

$Q_{1}$ is nonempty due to the definition of $M_{\partial} \cdot Q_{2}$ is nonempty since $I_{i_{0}}\left(t_{0}\right)>0, W_{i_{0}}\left(t_{0}\right)>0$. For any $i \in Q_{2}$ and we have that

$$
\frac{d W_{i}\left(t_{0}\right)}{d t_{0}}=k_{i} I_{i}\left(t_{0}\right)-\delta_{i} W_{i}\left(t_{0}\right)>k_{i} I_{i}\left(t_{0}\right), \quad i \in Q_{2} .
$$

It follows that there is an $\eta>0$ such that $I_{i}(t)>0$, for $t_{0}<$ $t<t_{0}+\eta, i \in Q_{2}$. This means that $\left(S_{i}(t), I_{i}(t), V_{i}(t), W_{i}(t), i=\right.$ $1,2, \ldots, n)$ does not belong to $\partial X_{0}$ for $t_{0}<t<t_{0}+\eta$, which contradicts the assumption that $\left(S_{i}(0), I_{i}(0), V_{i}(0), W_{i}(0), i=\right.$ $1,2, \ldots, n) \in M_{\partial}$. This proves the system (41).

$P_{0}$ is globally asymptotically stable for system (2). It is clear that there is only an equilibriaum $P_{0}$ in $M_{\partial}$ and by aforementioned claim, it then follows that $P_{0}$ is isolated invariant set in $X, W^{s}\left(P_{0}\right) \cap X_{0}=\emptyset$. Clearly, every orbit in $M_{\partial}$ converges to $P_{0}, P_{0}$ is acyclic in $M_{\partial}$. Using Theorem 4.6 in Thieme [26], we conclude that the system (2) is uniformly persistent with respect to $\left(X_{0}, \partial X_{0}\right)$. By the result of $[27,28]$, system (2) has an equilibrium $\left(S_{1}^{*}, I_{1}^{*}, V_{1}^{*}, W_{1}^{*}, \ldots, S_{n}^{*}, I_{n}^{*}, V_{n}^{*}, W_{n}^{*}\right) \in$ $X_{0}$. We further claim that $S_{i}^{*}, V_{i}^{*}>0, i=1,2, \ldots, n$. Suppose that $S_{i}^{*}=V_{i}^{*}=0, i=1,2, \ldots, n$; from of (2), we can get $I_{i}^{*}=W_{i}^{*}=0, i=1,2, \ldots, n$. It is a contradiction. Then $\left(S_{1}^{*}, I_{1}^{*}, V_{1}^{*}, W_{1}^{*}, \ldots, S_{n}^{*}, I_{n}^{*}, V_{n}^{*}, W_{n}^{*}\right) \in$ $X_{0}$ is a componentwise positive equilibrium of system (2). This completes the proof.

The following theorem shows that there exists a unique positive solution for system (2) when $\mathscr{R}_{0}>1$.

Theorem 4. If $\mathscr{R}_{0}>1$, then there only exists a unique positive equilibrium $P^{*}$ for system (2).

Proof. Consider the following system:

$$
\begin{aligned}
& A_{i}-\left(d_{i}+\gamma_{i}\right) S_{i}+\lambda_{i} V_{i}-\sum_{j=1}^{n} \beta_{i j} S_{i} W_{j}=0, \\
& \sum_{j=1}^{n} \beta_{i j} S_{i} W_{j}-\left(d_{i}+r_{i}\right) I_{i}=0,
\end{aligned}
$$




$$
\begin{aligned}
& \gamma_{i} S_{i}-\left(\lambda_{i}+d_{i}\right) V_{i}=0, \\
& k_{i} I_{i}-\delta_{i} W_{i}=0,
\end{aligned}
$$

$$
i=1,2, \ldots, n \text {. }
$$

We have that

$$
\begin{array}{r}
S_{i}=\frac{d_{i}+\lambda_{i}}{d_{i}\left(d_{i}+\lambda_{i}+\gamma_{i}\right)}\left(A_{i}-\left(d_{i}+r_{i}\right) I_{i}\right), \\
W_{i}=\frac{k_{i} I_{i}}{\delta_{i}}, \quad V_{i}=\frac{\gamma_{i} S_{i}}{d_{i}+\lambda_{i}}, \\
\quad i=1,2, \ldots, n .
\end{array}
$$

Hence, the equilibrium of system (2) is equal to the following system:

$$
B_{i}\left(A_{i}-n_{i} I_{i}\right) \sum_{j=1}^{n} \beta_{i j} I_{j}-n_{i} I_{i}=0, \quad i=1,2, \ldots, n,
$$

where

$$
B_{i}=\frac{k_{i}\left(d_{i}+\lambda_{i}\right)}{d_{i} \delta_{i}\left(d_{i}+\lambda_{i}+\gamma_{i}\right)}, \quad n_{i}=d_{i}+r_{i}, \quad i=1,2, \ldots, n .
$$

Therefore, we only need to prove that (46) has a unique positive equilibrium when $\mathscr{R}_{0}>1$. Use the method in [12] to demonstrate the unique positive equilibrium of (46). First we prove that $I_{i}^{*}=h, i=1,2, \ldots, n$, is the only positive solution of (46). Assume that $I_{i}^{*}=h$ and $I_{i}^{*}=$ $k$ are two positive solutions of (46), both nonzero. If $h \neq k$, then $h_{i} \neq k_{i}$ for some $i(i=1,2, \ldots, n)$. Assume without loss of generality that $h_{1}>k_{1}$ and moreover that $h_{1} / k_{1} \geq$ $h_{i} / k_{i}$ for all $i(i=1,2, \ldots, n)$. Since $h$ and $k$ are positive solutions of (46), we substitute them into (46). We obtain

$$
\begin{aligned}
0 & =B_{1}\left(A_{1}-n_{1} h_{1}\right) \sum_{j=1}^{n} \beta_{1 j} h_{j}-n_{1} h_{1} \\
& =B_{1}\left(A_{1}-n_{1} k_{1}\right) \sum_{j=1}^{n} \beta_{1 j} k_{j}-n_{1} k_{1},
\end{aligned}
$$

so

$$
\begin{gathered}
0=B_{1}\left(A_{1}-n_{1} h_{1}\right) \sum_{j=1}^{n} \beta_{1 j} h_{j} \frac{k_{1}}{h_{1}}-n_{1} k_{1} \\
=B_{1}\left(A_{1}-n_{1} k_{1}\right) \sum_{j=1}^{n} \beta_{1 j} k_{j}-n_{1} k_{1}, \\
B_{1}\left(A_{1}-n_{1} h_{1}\right) \sum_{j=1}^{n} \beta_{1 j} h_{j} \frac{k_{1}}{h_{1}}=B_{1}\left(A_{1}-n_{1} k_{1}\right) \sum_{j=1}^{n} \beta_{1 j} k_{j} .
\end{gathered}
$$

But $\left(h_{i} / h_{1}\right) k_{1} \leq k_{i}$ and $B_{1}\left(A_{1}-n_{1} h_{1}\right)<B_{1}\left(A_{1}-n_{1} k_{1}\right)$; thus from the above equalities we get

$$
\begin{aligned}
& B_{1}\left(A_{1}-n_{1} h_{1}\right) \sum_{j=1}^{n} \beta_{1 j} h_{j} \frac{k_{1}}{h_{1}} \\
& \leq B_{1}\left(A_{1}-n_{1} h_{1}\right) \sum_{j=1}^{n} \beta_{1 j} k_{j} \\
& <B_{1}\left(A_{1}-n_{1} k_{1}\right) \sum_{j=1}^{n} \beta_{1 j} k_{j} .
\end{aligned}
$$

This is a contradiction, so there is only one positive solution $I_{i}^{*}=h, i=1,2, \ldots, n$, of (46). So when $\mathscr{R}_{0}>1$, there only exists a unique positive equilibrium for system (2).

2.4. Global Stability of the Unique Endemic Solution of System (2). In this section, we prove that the unique endemic equilibrium of system (2) is globally asymptotically stable in $X_{0}$. In order to prove global stability of the endemic equilibrium, the Lyapunov function will be used. In the following, we also use a Lyapunov function to prove global stability of the endemic equilibrium.

Theorem 5. If $\mathscr{R}_{0}>1$, the unique positive equilibrium $P^{*}$ of system (2) is globally asymptotically stable in $X_{0}$.

Proof. Following [15] we define

$$
\begin{gathered}
\xi_{i j}=\beta_{i j} s_{i}^{*} W_{j}^{*}, \quad 1 \leq i, \quad j \leq n, n \geq 2, \\
\mathbf{B}=\left(\begin{array}{cccc}
\sum_{j \neq 1}^{n} \xi_{1 j} & -\xi_{21} & \cdots & -\xi_{n 1} \\
-\xi_{12} & \sum_{j \neq 2}^{n} \xi_{2 j} & \cdots & -\xi_{n 2} \\
\vdots & \vdots & \ddots & \vdots \\
-\xi_{1 n} & -\xi_{2 n} & \cdots & \sum_{j \neq n}^{n} \xi_{n j}
\end{array}\right)_{n \times n},
\end{gathered}
$$

which is a Laplacian matrix whose column sums are zero and which is irreducible. Therefore, it follows from Lemma 2.1 of [15] that the solution space of linear system

$$
\mathbf{B} \zeta=0,
$$

has dimension 1 with a basis

$$
\zeta=\left(\zeta_{1}, \zeta_{2}, \ldots, \zeta_{n}\right)^{T}=\left(c_{1}, c_{2}, \ldots, c_{n}\right)^{T},
$$

where $c_{i}$ denotes the cofactor of the $i$ th diagonal entry of $\mathbf{B}$. Note that from (53) we have that

$$
\sum_{j=1}^{n} \zeta_{i} \xi_{i j}=\sum_{j=1}^{n} \zeta_{j} \xi_{j i}, \quad i=1,2, \ldots, n
$$



tion

For such $\zeta=\left(\zeta_{1}, \zeta_{2}, \ldots, \zeta_{n}\right)$ we define a Lyapunov func-

$L(\mathbf{S}, \mathbf{I}, \mathbf{V}, \mathbf{W})$

$$
\begin{aligned}
=\sum_{i=1}^{n} \zeta_{i}( & S_{i}-S_{i}^{*}-S_{i}^{*} \ln \frac{S_{i}^{*}}{S_{i}}+I_{i}-I_{i}^{*}-I_{i}^{*} \ln \frac{I_{i}^{*}}{I_{i}} \\
& +V_{i}-V_{i}^{*}-V_{i}^{*} \ln \frac{V_{i}^{*}}{V_{i}} \\
& \left.+\frac{d_{i}+r_{i}}{k_{i}}\left(W_{i}-W_{i}^{*}-W_{i}^{*} \ln \frac{W_{i}^{*}}{W_{i}}\right)\right),
\end{aligned}
$$

where $\mathbf{S}=\left(S_{1}, S_{2}, \ldots, S_{n}\right), \mathbf{I}=\left(I_{1}, I_{2}, \ldots, I_{n}\right), \mathbf{V}=$ $\left(V_{1}, V_{2}, \ldots, V_{n}\right)$, and $\mathbf{W}=\left(W_{1}, W_{2}, \ldots, W_{n}\right)$. It is easy to see that $L(\mathbf{S}, \mathbf{I}, \mathbf{V}, \mathbf{W}) \geq 0$ for all $(\mathbf{S}, \mathbf{I}, \mathbf{V}, \mathbf{W}) \geq 0$ and the equality $L(\mathbf{S}, \mathbf{I}, \mathbf{V}, \mathbf{W})=0$ holds if and only if $(\mathbf{S}, \mathbf{I}, \mathbf{V}, \mathbf{W})=$ $\left(\mathbf{S}^{*}, \mathbf{I}^{*}, \mathbf{V}^{*}, \mathbf{W}^{*}\right)$. The derivative along the trajectories of system (2) is

$$
\begin{aligned}
& L^{\prime}(\mathbf{S}, \mathbf{I}, \mathbf{V}, \mathbf{W}) \\
& =\sum_{i=1}^{n} \zeta_{i}\left(A_{i}-\left(d_{i}+\gamma_{i}\right) S_{i}+\lambda_{i} V_{i}-\sum_{j=1}^{n} \beta_{i j} S_{i} W_{j}\right. \\
& -\frac{S_{i}^{*}}{S_{i}}\left(A_{i}-\left(d_{i}+\gamma_{i}\right) S_{i}+\lambda_{i} V_{i}-\sum_{j=1}^{n} \beta_{i j} S_{i} W_{j}\right) \\
& +\sum_{j=1}^{n} \beta_{i j} S_{i} W_{j}-\left(d_{i}+r_{i}\right) I_{i} \\
& -\frac{I_{i}^{*}}{I_{i}}\left(\sum_{j=1}^{n} \beta_{i j} S_{i} W_{j}-\left(d_{i}+r_{i}\right) I_{i}\right)+\gamma_{i} S_{i} \\
& -\left(\lambda_{i}+d_{i}\right) V_{i}-\frac{V_{i}^{*}}{V_{i}}\left(\gamma_{i} S_{i}-\left(\lambda_{i}+d_{i}\right) V_{i}\right) \\
& \left.+\frac{d_{i}+r_{i}}{k_{i}}\left(k_{i} I_{i}-\delta_{i} W_{i}-\frac{W_{i}^{*}}{W_{i}}\left(k_{i} I_{i}-\delta_{i} W_{i}\right)\right)\right) \\
& =L_{1}+L_{2}+L_{3} \text {. }
\end{aligned}
$$

From system (44), we have

$$
\begin{gathered}
A_{i}=\left(d_{i}+\gamma_{i}\right) S_{i}^{*}-\lambda_{i} V_{i}^{*}+\sum_{j=1}^{n} \beta_{i j} S_{i}^{*} W_{j}^{*}, \\
\sum_{j=1}^{n} \beta_{i j} S_{i}^{*} W_{j}^{*}=\left(d_{i}+r_{i}\right) I_{i}^{*}=\frac{\delta_{i}\left(d_{i}+r_{i}\right) W_{i}^{*}}{k_{i}} .
\end{gathered}
$$

$$
\begin{aligned}
& L_{1}=\sum_{i=1}^{n} \zeta_{i}\left(\sum_{j=1}^{n} \beta_{i j} S_{i}^{*} W_{j}-\frac{\delta_{i}\left(d_{i}+r_{i}\right) W_{i}}{k_{i}}\right), \\
& L_{2}=\sum_{i=1}^{n} \zeta_{i}\left(\left(d_{i}+\gamma_{i}\right) S_{i}^{*}-\lambda_{i} V_{i}^{*}-\left(d_{i}+\gamma_{i}\right) S_{i}+\lambda_{i} V_{i}\right. \\
& +\frac{S_{i}^{*}}{S_{i}}\left(\left(d_{i}+\gamma_{i}\right) S_{i}^{*}-\lambda_{i} V^{*}-\left(d_{i}+\gamma_{i}\right) S_{i}+\lambda_{i} V_{i}\right) \\
& +\gamma_{i} S_{i}-\left(\lambda_{i}+d_{i}\right) V_{i} \\
& \left.+\frac{V_{i}^{*}}{V_{i}}\left(\gamma_{i} S_{i}-\left(\lambda_{i}+d_{i}\right) V_{i}\right)\right) \\
& =\sum_{i=1}^{n} \zeta_{i}\left(d_{i} S_{i}^{*}\left(2-\frac{S_{i}}{S_{i}^{*}}-\frac{S_{i}^{*}}{S_{i}}\right)\right. \\
& +\lambda_{i} V_{i}^{*}\left(2-\frac{S_{i} V_{i}^{*}}{S_{i}^{*} V_{i}}-\frac{S_{i}^{*} V_{i}}{S_{i} V_{i}^{*}}\right) \\
& \left.+d_{i} V_{i}^{*}\left(3-\frac{V_{i}}{V_{i}^{*}}-\frac{S_{i}^{*}}{S_{i}}-\frac{S_{i} V_{i}^{*}}{S_{i}^{*} V_{i}}\right)\right) \leq 0, \\
& L_{3}=\sum_{i=1}^{n} \zeta_{i}\left(3 \sum_{j=1}^{n} \beta_{i j} S_{i}^{*} W_{j}^{*}-\sum_{j=1}^{n} \beta_{i j} S_{i}^{*} W_{j}^{*} \frac{S_{i}^{*}}{S_{i}}\right. \\
& \left.-\sum_{j=1}^{n} \beta_{i j} S_{i} W_{j} \frac{I_{i}^{*}}{I_{i}}-\left(d_{i}+r_{i}\right) I_{i} \frac{W_{i}^{*}}{W_{i}}\right) .
\end{aligned}
$$

Now we claim that

$$
\sum_{i=1}^{n} \zeta_{i} \sum_{j=1}^{n} \beta_{i j} S_{i}^{*} W_{j}=\sum_{i=1}^{n} \zeta_{i} \frac{\delta_{i}\left(d_{i}+r_{i}\right) W_{i}}{k_{i}}
$$

Appealing to (51), (55) and (59),

$$
\begin{aligned}
& \sum_{i=1}^{n} \sum_{j=1}^{n} \zeta_{i} \beta_{i j} S_{i}^{*} W_{j} \\
& =\sum_{i=1}^{n} \sum_{j=1}^{n} \zeta_{j} \beta_{j i} S_{j}^{*} W_{i}=\sum_{i=1}^{n} \sum_{j=1}^{n} \frac{W_{i}}{W_{i}^{*}} \zeta_{j} \beta_{j i} S_{j}^{*} W_{i}^{*} \\
& =\sum_{i=1}^{n} \frac{W_{i}}{W_{i}^{*}} \sum_{j=1}^{n} \zeta_{j} \xi_{j i}=\sum_{i=1}^{n} \frac{W_{i}}{W_{i}^{*}} \sum_{j=1}^{n} \zeta_{i} \zeta_{i j} \\
& =\sum_{i=1}^{n} \zeta_{i} \frac{\delta_{i}\left(d_{i}+r_{i}\right) W_{i}}{k_{i}} .
\end{aligned}
$$


From (61) we have

$L^{\prime}(\mathbf{S}, \mathbf{I}, \mathbf{V}, \mathbf{W})$

$$
\begin{aligned}
\leq & \sum_{i=1}^{n} \zeta_{i}\left(3 \sum_{j=1}^{n} \beta_{i j} S_{i}^{*} W_{j}^{*}-\sum_{j=1}^{n} \beta_{i j} S_{i}^{*} W_{j}^{*} \frac{S_{i}^{*}}{S_{i}}\right. \\
& \left.\quad-\sum_{j=1}^{n} \beta_{i j} S_{i} W_{j} \frac{I_{i}^{*}}{I_{i}}-\left(d_{i}+r_{i}\right) I_{i} \frac{W_{i}^{*}}{W_{i}}\right) \\
= & \sum_{i, j=1}^{n} \zeta_{i} \xi_{i j}\left(3-\frac{S_{i}^{*}}{S_{i}}-\frac{S_{i} W_{j} I_{i}^{*}}{S_{i}^{*} W_{j}^{*} I_{i}}-\frac{W_{i}^{*} I_{i}}{W_{i} I_{i}^{*}}\right) \\
= & : H_{n}\left(S_{1}, I_{1}, W_{1}, \ldots, S_{n}, I_{n}, W_{n}\right) .
\end{aligned}
$$

Next we show that $H_{n} \leq 0$ for all $\left(S_{1}, I_{1}, W_{1}, \ldots, S_{n}\right.$, $\left.I_{n}, W_{n}\right) \in X_{0}$ by applying the graph-theoretic approach developed in [29-31]. As in [29], $L=G(B)$ denotes the directed graph associated with matrix $\mathbf{B}, Q$ presents a subgraph of $L, C Q$ denotes the unique elementary cycle of $Q, E(C Q)$ presents the set of directed arcs in CQ, and $l=$ $l(Q)$ denotes the number of arcs in CQ. Then $H_{n}$ can be rewritten as

$$
H_{n}=\sum_{Q} H_{n, Q}
$$

where

$$
\begin{aligned}
H_{n, \mathrm{Q}}= & \prod_{(r, m) \in E(\mathrm{Q})} \xi_{r m} \\
& \times\left(3 l-\sum_{(i, j) \in E(\mathrm{CQ})}\left(\frac{S_{i}^{*}}{S_{i}}+\frac{S_{i} W_{j} I_{i}^{*}}{S_{i}^{*} W_{j}^{*} I_{i}}+\frac{W_{i}^{*} I_{i}}{W_{i} I_{i}^{*}}\right)\right) .
\end{aligned}
$$

For instance,

$$
\begin{aligned}
H_{1}= & H_{1}\left(S_{1}, I_{1}, W_{1}\right) \\
= & \sum_{i=j=1} \zeta_{1} \xi_{11}\left(3-\frac{S_{1}^{*}}{S_{1}}-\frac{S_{1} W_{1} I_{1}^{*}}{S_{1}^{*} W_{1}^{*} I_{1}}-\frac{W_{1}^{*} I_{1}}{W_{1} I_{1}^{*}}\right) \leq 0, \\
H_{2}= & H_{2}\left(S_{1}, I_{1}, W_{1}, S_{2}, I_{2}, W_{2}\right) \\
= & \sum_{i, j=1}^{2} \zeta_{i} \xi_{i j}\left(3-\frac{S_{i}^{*}}{S_{i}}-\frac{S_{i} W_{j} I_{i}^{*}}{S_{i}^{*} W_{j}^{*} I_{i}}-\frac{W_{i}^{*} I_{i}}{W_{j} I_{i}^{*}}\right) \\
= & \xi_{11} \xi_{21}\left(3-\frac{S_{1}^{*}}{S_{1}}-\frac{S_{1} W_{1} I_{1}^{*}}{S_{1}^{*} W_{1}^{*} I_{1}}-\frac{W_{1}^{*} I_{1}}{W_{1} I_{1}^{*}}\right) \\
& +\xi_{22} \xi_{12}\left(3-\frac{S_{2}^{*}}{S_{2}}-\frac{S_{2} W_{2} I_{2}^{*}}{S_{2}^{*} W_{2}^{*} I_{2}}-\frac{W_{2}^{*} I_{2}}{W_{2} I_{2}^{*}}\right) \\
& +\xi_{12} \xi_{21}\left(6-\frac{S_{1}^{*}}{S_{1}}-\frac{S_{1} W_{1} I_{1}^{*}}{S_{1}^{*} W_{1}^{*} I_{1}}-\frac{W_{1}^{*} I_{1}}{W_{1} I_{1}^{*}}-\frac{S_{2}^{*}}{S_{2}}\right. \\
& \left.-\frac{S_{2} W_{2} I_{2}^{*}}{S_{2}^{*} W_{2}^{*} I_{2}}-\frac{W_{2}^{*} I_{2}}{W_{2} I_{2}^{*}}\right) \leq 0 .
\end{aligned}
$$

Note that for each unicycle graph $Q$, it is easy to see that

$$
\prod_{(i, j) \in E(C Q)} \frac{S_{i}^{*}}{S_{i}} \cdot \frac{S_{i} W_{j} I_{i}^{*}}{S_{i}^{*} W_{j}^{*} I_{i}} \cdot \frac{W_{i}^{*} I_{i}}{W_{i} I_{i}^{*}}=\prod_{(i, j) \in E(C Q)} \frac{W_{i}^{*} W_{j}}{W_{i} W_{j}^{*}}=1 .
$$

Therefore,

$$
\sum_{(i, j) \in E(C Q)}\left(\frac{S_{i}^{*}}{S_{i}}+\frac{S_{i} W_{j} I_{i}^{*}}{S_{i}^{*} W_{j}^{*} I_{i}}+\frac{W_{i}^{*} I_{i}}{W_{i} I_{i}^{*}}\right) \geq 3 l
$$

and hence $H_{n, \mathrm{Q}} \leq 0$ for each $Q$, and $H_{n, \mathrm{Q}}=0$ if and only if

$$
\frac{S_{i}^{*}}{S_{i}}=\frac{S_{i} W_{j} I_{i}^{*}}{S_{i}^{*} W_{j}^{*} I_{i}}=\frac{W_{i}^{*} I_{i}}{W_{i} I_{i}^{*}}, \quad(i, j) \in E(C Q) .
$$

Thus

$$
L^{\prime}(\mathbf{S}, \mathbf{I}, \mathbf{V}, \mathbf{W}) \leq H_{n} \leq 0
$$

The equality $L^{\prime}(\mathbf{S}, \mathbf{I}, \mathbf{V}, \mathbf{W})=0$ holds if and only if $S_{i}=$ $S_{i}^{*}, I_{i}=I_{i}^{*}, V_{i}=V_{i}^{*}$, and $W_{i}=W_{i}^{*}$ for all $i=1,2, \ldots, n$. Therefore, following from LaSalle's Invariance Principle [32], the unique endemic equilibrium $P^{*}$ of system (2) is globally asymptotically stable. This completes the proof.

\section{A Numerical Example}

Consider the system (1), when $i=2$, one has the two-group model as follows:

$$
\begin{gathered}
\frac{d S_{1}}{d t}=A_{1}-\left(d_{1}+\gamma_{1}\right) S_{1}+\lambda_{1} V_{1}-\left(\beta_{11} S_{1} W_{1}+\beta_{12} S_{1} W_{2}\right), \\
\frac{d I_{1}}{d t}=\beta_{11} S_{1} W_{1}+\beta_{12} S_{1} W_{2}-\left(d_{1}+r_{1}\right) I_{1}, \\
\frac{d R_{1}}{d t}=r_{1} I_{i}-d_{1} R_{1}, \\
\frac{d V_{1}}{d t}=\gamma_{1} S_{1}-\left(\lambda_{1}+d_{1}\right) V_{1}, \\
\frac{d W_{1}}{d t}=k_{1} I_{1}-\delta_{1} W_{1}, \\
\frac{d S_{2}}{d t}=A_{2}-\left(\begin{array}{c}
d 2 \\
\left.+\gamma_{2}\right) S_{2}+\lambda_{2} V_{2}-\left(\beta_{21} S_{2} W_{1}+\beta_{22} S_{2} W_{2}\right), \\
\frac{d I_{2}}{d t}=\beta_{21} S_{2} W_{1}+\beta_{22} S_{2} W_{2}-\left(d_{2}+r_{2}\right) I_{2}, \\
\frac{d R_{2}}{d t}=r_{2} I_{2}-d_{2} R_{2}, \\
\frac{d V_{2}}{d t}=\gamma_{2} S_{2}-\left(\lambda_{2}+d_{2}\right) V_{2}, \\
d t
\end{array}=k_{2} I_{2}-\delta_{2} W_{2} .\right.
\end{gathered}
$$


We can give the basic reproduction number of system (71), which is

$$
\mathscr{R}_{0}^{\prime}=\frac{A_{11}+A_{22}+\sqrt{\left(A_{11}-A_{22}\right)^{2}+4 A_{12} A_{21}}}{2},
$$

where

$$
A_{i j}=\frac{\beta_{i j} k_{j} S_{i}^{0}}{\delta_{j}\left(d_{j}+r_{j}\right)}, \quad S_{i}^{0}=\frac{A_{i}\left(\lambda_{i}+d_{i}\right)}{d_{i}\left(\lambda_{i}+d_{i}+\gamma_{i}\right)}, \quad i=1,2 .
$$

Taking $A_{1}=150, A_{2}=220, d_{1}=0.1, d_{2}=0.1, \lambda_{1}=0.4$, $\lambda_{2}=0.6, \lambda_{1}=0.5, \lambda_{2}=0.5, r_{1}=1, r_{2}=1, k_{1}=10$, $k_{2}=10, \delta_{1}=8, \delta_{2}=8$ and using Matlab ODE solver, we run numerical simulations for two cases.

If $\beta_{11}=0.00048, \beta_{12}=0.0004, \beta_{21}=0.0004$, and $\beta_{22}=$ 0.00045 , we have $\mathscr{R}_{0}^{\prime} \approx 0.9804<1$. Hence the disease-free equilibrium of system (71) is globally asymptotically stable (see Figure 1(a)). If $\beta_{11}=0.0025, \beta_{12}=0.001, \beta_{21}=0.001$, and $\beta_{22}=0.0020$, we have $\mathscr{R}_{0}^{\prime} \approx 3.6594>1$. Hence the endemic equilibrium of system (71) is globally asymptotically stable (see Figure 1(b)).

\section{Conclusion}

Cholera epidemic has become a major health problem for many developing countries. From good understanding of the transmission dynamics of cholera in many emergent epidemic regions, the heterogeneous host population can be divided into several homogeneous groups according to modes of transmission, contact patterns, or geographic distributions. Hence, in this paper, we proposed a multigroup cholera SIRVW epidemiological model. In order to distinguish many multi-group models with direct transmission from person to person, we only considered this multigroup cholera model with indirect transmission from the bacteria of the aquatic environment to person. Firstly, the basic reproduction number $\mathscr{R}_{0}$ of this model is given. Then, it is found that the model has two non-negative equilibria, the disease-free equilibrium and the endemic equilibrium. The disease-free equilibrium exists without any condition whereas the endemic equilibrium exists provided $\mathscr{R}_{0}>1$. Finally, through the analysis of the model it has been found that the global asymptotic behavior of multi-group SIRVW model is completely determined by the size of $\mathscr{R}_{0}$. That is, the disease-free equilibrium is globally asymptotically stable if $\mathscr{R}_{0}<1$ while an endemic equilibrium exists uniquely and is globally asymptotically stable if $\mathscr{R}_{0}>1$. By running numerical simulations for the cases of two-groups model, we can see that the disease-free equilibrium of system (71) is globally stable when $\mathscr{R}_{0}^{\prime}<1$ and the unique endemic equilibrium of system (71) is globally stable when $\mathscr{R}_{0}^{\prime}>1$.

\section{Acknowledgments}

This research is supported by the National Natural Science Foundation of China under Grants 11301490, 11301491, 11331009, 11171314, and 11147015, Natural Science Foundation

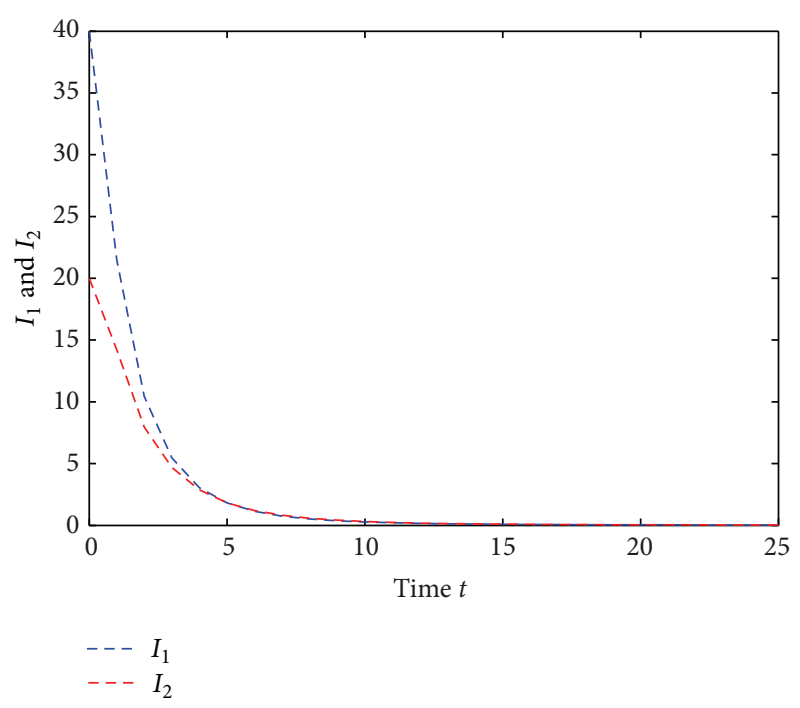

(a)

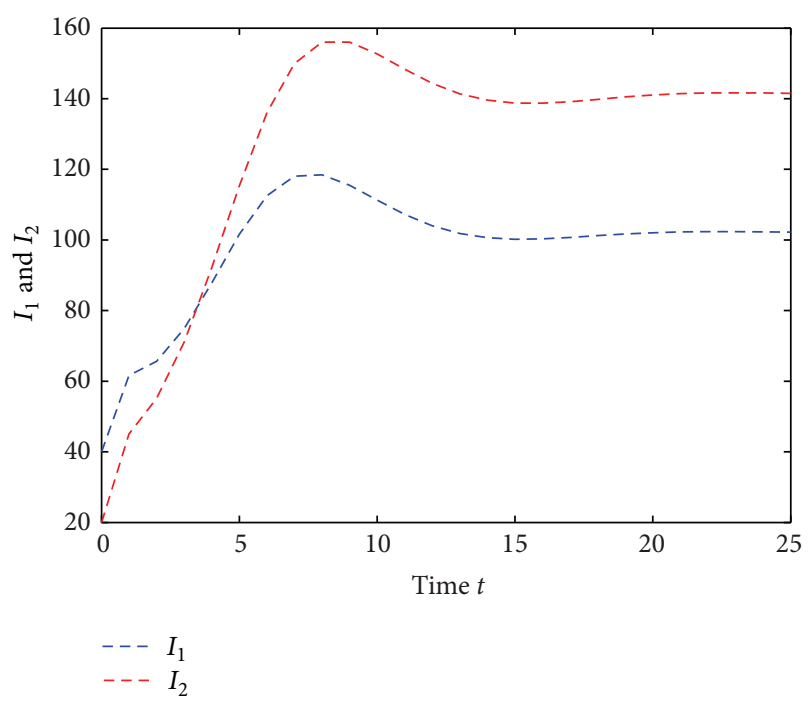

(b)

Figure 1: (a) The disease dies out in both groups. (b) The disease persists in both groups. Initial conditions are: $S_{1}(0)=280, I_{1}(0)=$ $40, R_{1}(0)=10, V_{1}(0)=130, W_{1}(0)=250, S_{2}(0)=260, I_{2}(0)=20$, $R_{2}(0)=10, V_{2}(0)=130, W_{2}(0)=300$.

of Shan'Xi Province Grant no. 2012021002-1, the specialized research fund for the doctoral program of higher education preferential development, no. 20121420130001, China Postdoctoral Science Foundation under Grant no. 2012M520814, Shanghai Postdoctoral Science Foundation under Grants no. 13R21410100 and IDRC104519-010.

\section{References}

[1] M. A. Jensen, S. M. Faruque, J. J. Mekalanos, and B. R. Levin, "Modeling the role of bacteriophage in the control of cholera outbreaks," Proceedings of the National Academy of Sciences of the United States of America, vol. 103, no. 12, pp. 4652-4657, 2006. 
[2] A. K. Misra and V. Singh, "A delay mathematical model for the spread and control of water borne diseases," Journal of Theoretical Biology, vol. 301, pp. 49-56, 2012.

[3] C. Torres Codeço, "Endemic and epidemic dynamics of cholera: the role of the aquatic reservoir," BMC Infectious Diseases, vol. 1 , article 1, 2001.

[4] M. Pascual, M. J. Bouma, and A. P. Dobson, "Cholera and climate: revisiting the quantitative evidence," Microbes and Infection, vol. 4, no. 2, pp. 237-245, 2002.

[5] D. M. Hartley, J. G. Morris Jr., and D. L. Smith, "Hyperinfectivity: a critical element in the ability of V. cholerae to cause epidemics?" PLoS Medicine, vol. 3, no. 1, pp. 63-69, 2006.

[6] Z. Mukandavire, S. Liao, J. Wang, H. Gaff, D. L. Smith, and J. G. Morris Jr., "Estimating the reproductive numbers for the 2008-2009 cholera outbreaks in Zimbabwe," Proceedings of the National Academy of Sciences of the United States of America, vol. 108, no. 21, pp. 8767-8772, 2011.

[7] Z. Mukandavire, D. L. Smith, and J. G. Morris Jr., "Cholera in Haiti: reproductive numbers and vaccination coverage estimates," Scientific Reports, vol. 3, article 997, 2013.

[8] W. Z. Huang, K. L. Cooke, and C. Castillo-Chavez, "Stability and bifurcation for a multiple-group model for the dynamics of HIV/AIDS transmission," SIAM Journal on Applied Mathematics, vol. 52, no. 3, pp. 835-854, 1992.

[9] Z. Feng and J. X. Velasco-Hernández, "Competitive exclusion in a vector-host model for the dengue fever," Journal of Mathematical Biology, vol. 35, no. 5, pp. 523-544, 1997.

[10] C. Bowman, A. B. Gumel, P. Van den Driessche, J. Wu, and H. Zhu, "A mathematical model for assessing control strategies against West Nile virus," Bulletin of Mathematical Biology, vol. 67, pp. 1107-1133, 2005.

[11] R. Edwards, S. Kim, and P. van den Driessche, "A multigroup model for a heterosexually transmitted disease," Mathematical Biosciences, vol. 224, pp. 87-94, 2010.

[12] A. Lajmanovich and J. A. York, "A deterministic model for gonorrhea in a nonhomogeneous population," Mathematical Biosciences, vol. 28, pp. 221-236, 1976.

[13] H. W. Hethcote, "An immunization model for a heterogeneous population," Theoretical Population Biology, vol. 14, no. 3, pp. 338-349, 1978.

[14] H. R. Thieme, "Local stability in epidemic models for heterogeneous populations," in Mathematics in Biology and Medicine, V. Capasso, E. Grosso, and S. L. Paveri-Fontana, Eds., vol. 57 of Lecture Notes in Biomathematics, pp. 185-189, Springer, 1985.

[15] H. Guo, M. Y. Li, and Z. Shuai, "Global stability of the endemic equilibrium of multigroup SIR epidemic models," Canadian Applied Mathematics Quarterly, vol. 14, pp. 259-284, 2006.

[16] Z. Yuan and L. Wang, "Global stability of epidemiological models with group mixing and nonlinear incidence rates," Nonlinear Analysis. Real World Applications, vol. 11, no. 2, pp. 995-1004, 2010.

[17] R. Sun and J. Shi, "Global stability of multigroup epidemic model with group mixing and nonlinear incidence rates," Applied Mathematics and Computation, vol. 218, pp. 280-286, 2011.

[18] M. Y. Li, Z. Shuai, and C. Wang, "Global stability of multi-group epidemic models with distributed delays," Journal of Mathematical Analysis and Applications, vol. 361, pp. 38-47, 2010.

[19] H. Shu, D. Fan, and J. Wei, "Global stability of multi-group SEIR epidemic models with distributed delays and nonlinear transmission," Nonlinear Analysis. Real World Applications, vol. 13, no. 4, pp. 1581-1592, 2012.
[20] O. Diekmann, J. A. Heesterbeek, and J. A. Metz, "On the definition and the computation of the basic reproduction ratio R0 in models for infectious diseases in heterogeneous populations," Journal of Mathematical Biology, vol. 28, no. 4, pp. 365-382, 1990.

[21] P. van den Driessche and J. Watmough, "Reproduction numbers and sub-threshold endemic equilibria for compartmental models of disease transmission," Mathematical Biosciences, vol. 180, pp. 29-48, 2002.

[22] O. Diekmann, J. A. P. Heesterbeek, and M. G. Roberts, "The construction of next-generation matrices for compartmental epidemic models," Journal of the Royal Society Interface, vol. 7, no. 47 , pp. 873-885, 2010.

[23] H. L. Smith and P. Waltman, The Theory of the Chemostat, Cambridge University Press, 1995.

[24] H.R. Thieme, "Convergence results and a Poincaré-Bendixson trichotomy for asymptotically autonomous differential equations," Journal of Mathematical Biology, vol. 30, pp. 755-763, 1992.

[25] X. Q. Zhao and Z. J. Jing, "Global asymptotic behavior in some cooperative systems of functional differential equations," Canadian Applied Mathematics Quarterly, vol. 4, pp. 421-444, 1996.

[26] H. R. Thieme, "Persistence under relaxed point-dissipativity (with application to an endemic model)," Mathematical Biosciences, vol. 166, pp. 407-435, 1993.

[27] X. Q. Zhao, "Uniform persistence and periodic coexistence states in infinitedimensional periodic semiflows with applications," Canadian Applied Mathematics Quarterly, vol. 3, pp. 473495, 1995.

[28] W. D. Wang and X.-Q. Zhao, "An epidemic model in a patchy environment," Mathematical Biosciences, vol. 190, no. 1, pp. 97112,2004

[29] H. Guo, M. Y. Li, and Z. Shuai, "A graph-theoretic approach to the method of global Lyapunov functions," Proceedings of the American Mathematical Society, vol. 136, no. 8, pp. 2793-2802, 2008.

[30] J. W. Moon, Counting Labelled Trees, Canadian Mathematical Congress, Montreal, Canada, 1970.

[31] D. E. Knuth, The Art of Computer Programming, vol. 1, AddisonWesley, Reading, Mass, USA, 1997.

[32] J. P. Lasalle, “The stability of dynamical systems," in Proceedings of the Regional Conference Series in Applied Mathematics, SIAM, Philadelphia, Pa, USA, 1976. 


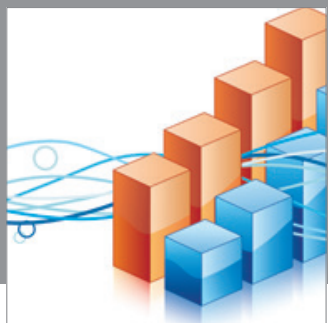

Advances in

Operations Research

mansans

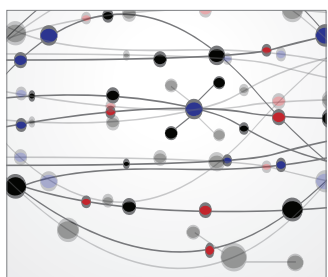

The Scientific World Journal
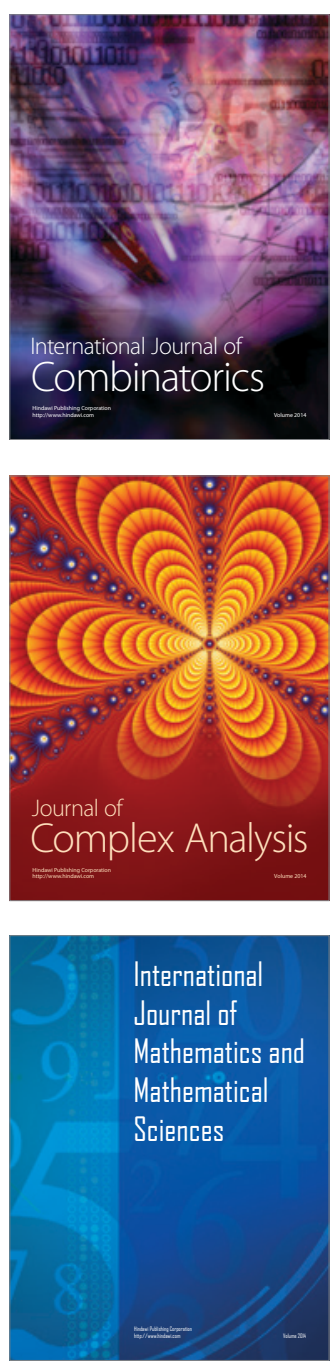
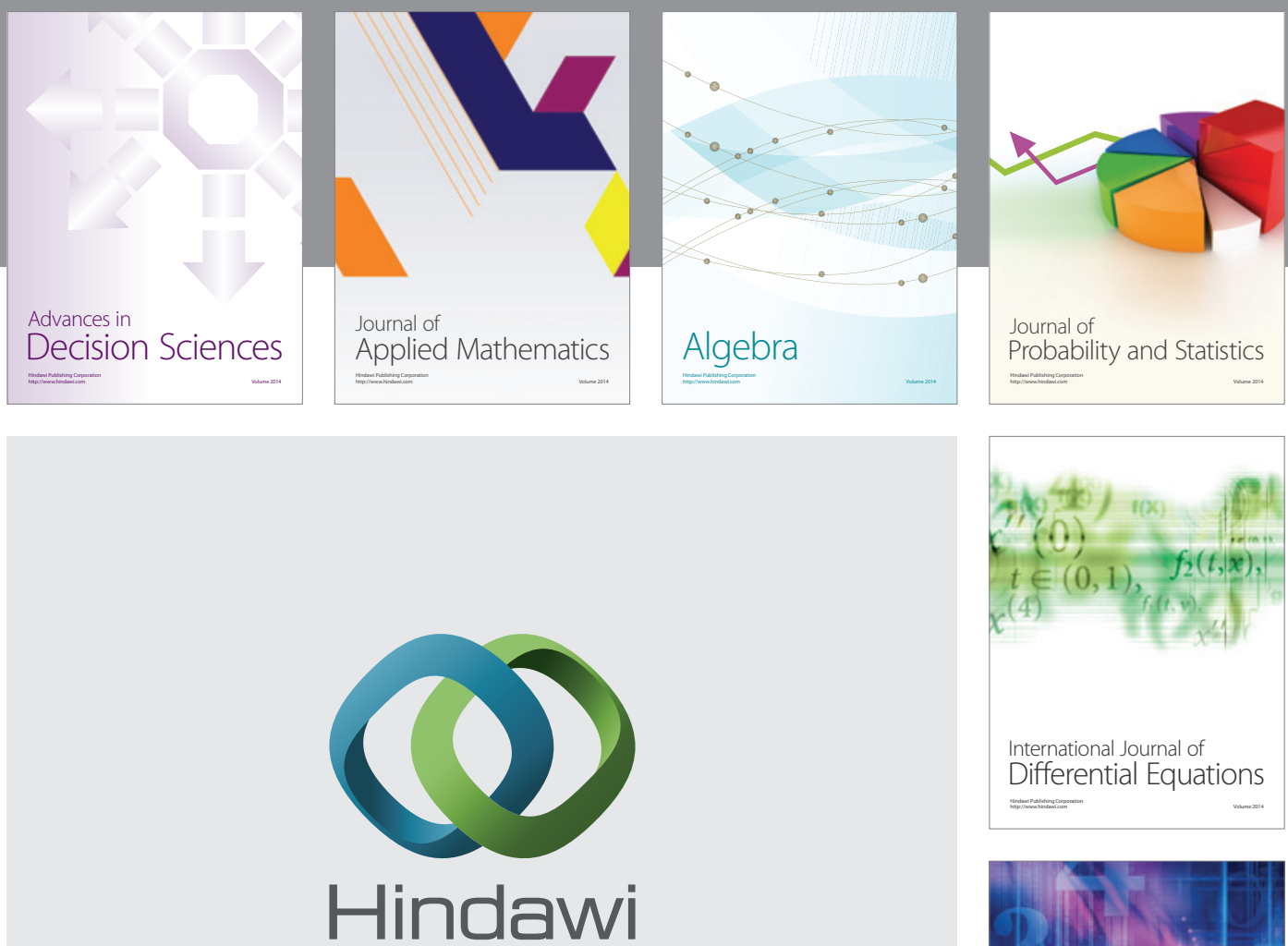

Submit your manuscripts at http://www.hindawi.com
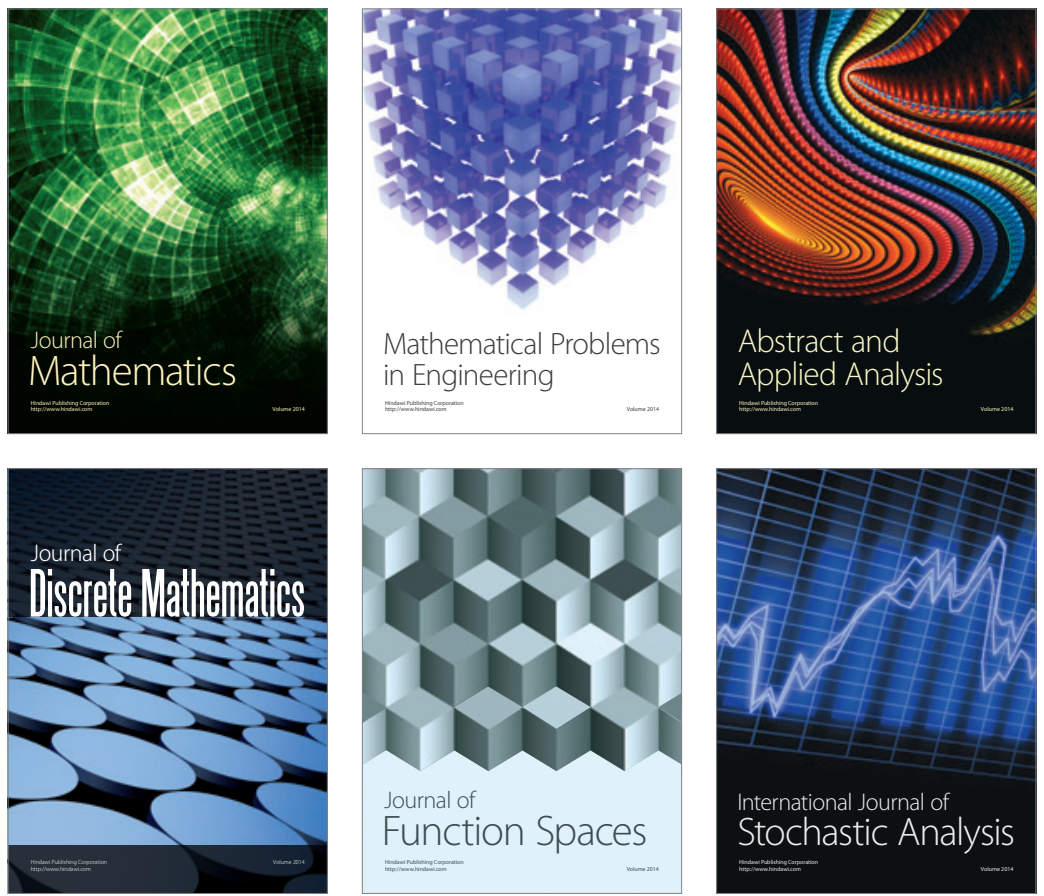

Journal of

Function Spaces

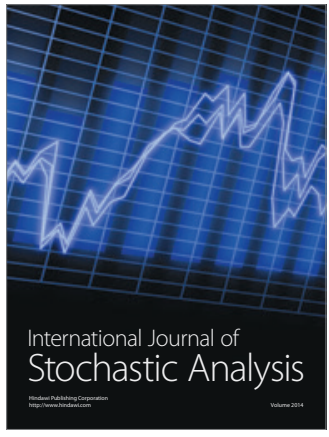

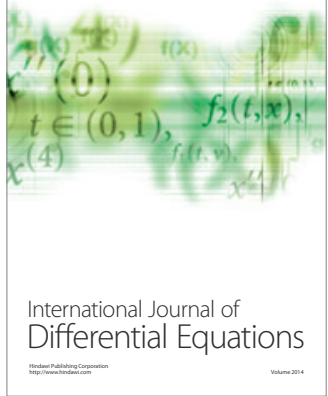
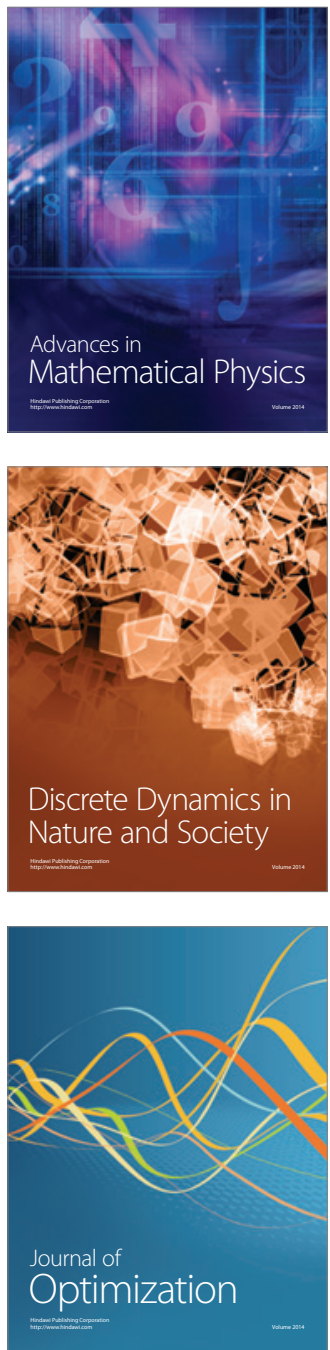